\title{
ESPECIE NUEVA DE CANGREJO DE ARENA DEL GÉNERO LEPIDOPA EN VERACRUZ, MÉXICO, Y UNA CLAVE ILUSTRADA DE LAS ESPECIES CITADAS PARA EL GOLFO DE MÉXICO Y EL MAR CARIBE
}

\section{A new species of sand crab of the genus Lepidopa from Veracruz, Mexico, and an illustrated key for the recorded species of the Gulf of Mexico and the Caribbean Sea}

\author{
Manuel Ortiz ${ }^{1}$, José Luis Tello-Musi² y Alberto Morales-Moreno²
}

${ }^{1}$ Laboratorio de Crustáceos, Facultad de Estudios Superiores Iztacala, Universidad Nacional Autónoma de México. ortiztouzet@yahoo.com.

${ }^{2}$ Laboratorio de Zoología, Facultad de Estudios Superiores Iztacala, Universidad Nacional Autónoma de México. jltm@unam.mx,amm@unam.mx.

\section{RESUMEN}

Se presenta la descripción de una especie nueva de cangrejo de arena del género Lepidopa. Ha sido colectada en la zona litoral de la playa Villa Rica, Veracruz, México. Existen 14 especies conocidas del género. De ellas, seis han sido citadas para el golfo de México y el mar Caribe. La nueva especie, Lepidopa boykoi, se distingue con facilidad de las restantes conocidas, al presentar sus pedúnculos oculares rectangulares, con sus órbitas situadas en los ángulos basales internos de los mismos. Se ofrece además una clave ilustrada para facilitar la identificación de las especies presentes en la región.

Palabras clave: cangrejo de arena, Lepidopa, especie nueva, Veracruz, México.

\section{ABSTRACT}

The description of a new species of the sand crab genus Lepidopa is presented. It has been collected in the litoral zone of the Villa Rica Beach, Veracruz, Mexico. There are 14 known species of the genus. Of these, six have been recorded for the Gulf of Mexico and the Caribbean Sea. The new species Lepidopa boykoi is easily distinguished from the other known by presenting its rectangular eye peduncles, with their orbits located at the internal basal angles thereof. It also offers an illustrated key for the identification of the species in the region.

Keywords: sand crab, Lepidopa, new species, Veracruz, Mexico.

\section{INTRODUCCIÓN}

Los cangrejos de arena son habitantes frecuentes de los fondos litorales y sublitorales someros de los mares tropicales. Se trata de anomuros simétricos, con su cuerpo deprimido dorso-ventralmente, que poseen su primer par de pereópodos sub-quelado (albuneidos) o simples (hípidos) y, como sucede en todos los anomuros, su quinto par de pereópodos aparece reducido. La creencia de que sus largas anténulas son empleadas para filtrar su alimento, tiende a ser descartada.

Para más detalles sobre estos anomuros u otros crustáceos decápodos de México y regiones adyacentes, el interesado podrá consultar la revisión mundial de los cangrejos de arena fósiles y recientes de las familias Albuneidae y Blepharipodidae de Boyko (2002), así como las especies de Cuba (Ortiz, et al., 2013; Ortiz, 2015) y la compilación de Álvarez et al. (2014). 
El género Lepidopa está constituido por 14 especies. De ellas, L. venusta Stimpson, 1859; L. richmondi Bendict, 1903; L. websteri Benedict, 1903; L. benedicti Schmitt, 1935; L. dexterae Abele y Efford, 1972 y L. luciae Boyko, 2002, aparecen representadas en numerosas localidades del golfo de México y el mar Caribe (Rodríguez, 1980; Boyko, 2002; Felder et al., 2009; WoRMS, 2018).

\section{OBJETIVO}

-Describir una especie nueva de cangrejo de arena del género Lepidopa y ofrecer una clave ilustrada original para la identificación de las especies presentes en la región.

\section{MATERIALES Y MÉTODOS}

La especie estudiada ha sido colectada en la zona litoral de forma manual. Todos los taxones que se presentan han sido validados con Boyko (2002) y WoRMS (2018). Los surcos del carapacho, CG 1-11, así como las partes del dactilo de un pereópodo, se describen de acuerdo con el patrón propuesto por Boyko (2002; Fig. 1). La clave que se presenta ha sido confeccionada considerando caracteres externos fáciles de observar. Las figuras han sido confeccionadas con el auxilio de una cámara clara y se han entintado con el programa Corel Draw-X4.

\section{RESULTADOS}

\section{TAXONOMÍA}

Phylum ARTHROPODA von Siebold, 1848

Subphylum CRUSTACEA Brünnich, 1772

Superclase MULTICRUSTACEA Regier, Schultz, Zwick,

Hussey, Ball. Wetzer, Martin, Cunningham, 2010

Clase MALACOSTRACA Latreille, 1802

Subclase EUMALACOSTRACA Latreille, 1802

Superorden EUCARIDA Calman, 1904

Orden DECAPODA Latreille, 1802

Superorden EUCARIDA Calman, 1904

Suberorden PLEOCYEMATA Burkenroad, 1863

Infraorden ANOMURA Mac Leay, 1838

Superfamilia HIPPOIDEA Latreille, 1825

Familia ALBUNEIDAE Stimpson, 1858

Subfamilia LEPIDOPINAE Boyko, 2002

Género Lepidopa Stimpson, 1858

Lepidopa boykoi sp. nov.

(Figs. 1-5)

Diagnosis. Margen anterior del carapacho con el rostro atrasado en relación a las espinas intermedias; pedúnculos oculares rectangulares; córneas dispuestas en el ángulo basal interno de sus pedúnculos; superficie anterior del carapacho con los campos setosos, CG-1, reducido a una estrecha franja dispuesta detrás del rostro, cóncava en su zona media y unida a los elementos medios y laterales, CG-2; dactilo del segundo pereópodo con el tacón redondeado; segmento pleonal II con las pleuras expandidas lateralmente; segmento pleonal VI es 1.1x más ancho que largo; margen posterior convexo; telson acorazonado. 
Diagnosis (in English). Anterior margin of the carapace with the face behind in relation to the intermediate thorns; rectangular eye peduncles; corneas located in the internal basal angle of their peduncles; anterior surface of the carapace with the setal field CG-1, reduced to a narrow stripe arranged behind the anterior margin, concave in its middle zone and joined to the middle and lateral elements, CG-2; dactyl of the second pereopod with rounded heel; pleonal segment II with laterally expanded pleura; pleonal segment VI 1.1x wider than long; with convex posterior margin; Heart-shaped telson.

\section{DESCRIPCIÓN}

\section{Holotipo, Hembra}

Carapacho. Aplanado; margen anterior con la punta del rostro retrasada en relación a las espinas intermedias; 1.1 x más ancho que largo, ancho máximo en su extremo anterior; divergiendo hacia su extremo posterior; margen anterior desprovisto de setas; senos oculares lisos y cóncavos; márgenes laterales con dos espinas dirigidas hacia delante; placa ocular pequeña y artejo peduncular intermedio, ambos no visibles en vista dorsal y situados bajo el rostro; rostro romo y corto, atrasado en relación a las espinas intermedias anteriores del carapacho; campo setoso CG-1, reducido a una estrecha franja dispuesta detrás del rostro, cóncava en su zona media y unida a los componentes medios y laterales; CG-2 ausente; CG-3 representado por dos surcos oblicuos y cortos; CG-4 ausente; CG-5 ausente; CG-6 estrecho, transverso y cóncavo; CG-7 a ambos lados y unidos a CG-6; formado por dos elementos oblicuos centrales algo separados; CG-8 elementos ligeramente marcados por dos zonas punteadas; CG-9 ausente; CG-10 formando dos elementos oblicuos cortos; CG-11 ausente; región hepática con surco oblicuo y espina lateral aguda; región cardíaca lisa (Figs. 1A, 2B).

Anténula. Artejos y flagelo desprovistos de setas largas; artejo uno ensanchado; artejo tres triangular; artejo tres el más corto y estrecho; endópodo pequeño, con dos artejos basales más largos que los tres distales; exópodo muy alargado, con 98 artejos (Figs. 2A, 2B).

Antena. Artejo tres triangular; artejo cuatro cuadrangular; artejo cinco del largo del cuatro, $0.3 \mathrm{x}$ el ancho del cuatro, con una seta distal muy larga; flagelo con ocho artejos; artejo uno con una seta ventral y otra dorsal; artejo dos con una seta dorsal y dos setas ventrales; artejo tres con una seta dorsal y otra ventral; artejo cuatro con una seta dorsal y dos ventrales; artejo cinco con un seta corta dorsal y dos ventrales; artejo seis con dos setas cortas dorsales; artejo siete con tres setas dorsales; artejo ocho $1.3 \mathrm{x}$ más largo que el siete, con dos setas distales (Figs. 2A, 2B).

Mandíbula. Proceso incisivo con dos dientes; borde cortante sin dientes; palpo con tres artejos semejantes en largo; artejo uno con nueve setas en su margen superior; artejo dos con nueve setas en su margen superior y 14 setas en su margen inferior; artejo tres con 23 setas recubriendo gran parte de sus márgenes (Fig. 3A).

Maxílula. Endito (no dibujado), cuadrangular, con setas marginales; exópodo con sus márgenes laterales paralelos; con 23 setas distales; endópodo proximal algo ensanchado distalmente; con 17 setas largas terminales con 15 más cortas insertadas entre las primeras. Lóbulo del endópodo truncado (Fig. 3B).

Maxila. Exópodo redondeado, con cuatro setas distales cortas y 14 más largas; escafognatito (no dibujado), formando un lóbulo redondeado posterior cubierto de setas cortas (Fig. 3C). 
Maxilípedo I. Epipodito (no dibujado), alargado, sin setas marginales; endópodo tan largo como el artejo distal del exopodo; con setas largas en su margen interno; exopodito con dos artejos; artejo uno tan largo como el dos; con ocho setas largas en su margen interno y 31 en el externo (Fig. 3D).

Maxilipedo II. Dactilo redondeado, cubierto de setas cortas marginales; propodio formando un discreto lóbulo dorsodistal; con siete setas cortas y ocho largas en su margen externo; carpo semejante en largo al propodio; con siete setas en su margen externo; mero alargado, con más de 30 setas en su margen interno y 18 en el externo; exopodito del largo del mero; artejo uno 3x del largo del dos (Fig. 3E).

Maxilípedo III. Dactilo alargado y con márgenes setosos, $0.5 \mathrm{x}$ del largo del propodio; margen interno del propodio protegido por el lóbulo anterior del carpo; con tres setas subterminales; carpo con 31 setas en su margen interno; mero tan largo como el carpo si se mide con su lóbulo anterior interno, con 10 setas en la mitad distal de su margen interno; exopodito corto con un solo artejo (Fig. 3F).

Pereópodo I derecho. Dactilo curvo, estrechándose gradualmente hacia su ápice; con una hilera de setas en su cara anterior; cara externa del propodio comprimida lateralmente, con sus caras laterales con grupos de setas muy cortas que no sobresalen; margen dorsal con una serie de setas alargadas; margen ventral desnudo; diente del ángulo palmar saliente, romo y con un penacho de setas distales; dedo móvil se entrecruza por fuera del mencionado diente; carpo con el ángulo dorso distal redondeado, inflado, tan alto como el del propodio; con tres hileras de setas paralelas; mero corto, $0.4 \mathrm{x}$ del largo del propodio (Figs. 4A, 4B).

Pereópodo I izquierdo. Dactilo y propodio semejantes a los del lado derecho; "dedo fijo" menos saliente que en el pereópodo del lado derecho; carpo menos setoso que en el del lado derecho; $0.3 \mathrm{x}$ del largo del propodio; con una pequeña cresta setosa en el punto medio de su borde dorsal; mero corto y semejante al del pereópodo derecho (Fig. 4C).

Pereópodo II. Dactilo con el tacón redondeado, margen base-tacón convexo, con setas cortas; base con tres penachos pequeños de setas marginales; escotadura aguda; margen inferior densamente setoso; punta ancha, plano convexa; propodio $0.7 \mathrm{x}$ el ancho del largo; carpo $2 \mathrm{x}$ más largo que ancho; con setas en su margen dorsal; mero1.2 x el largo del carpo; 2x más largo que ancho; con setas largas en ambos márgenes (Fig. 4D).

Pereópodo III. Dactilo con el tacón estrecho y saliente; margen base-tacón muy cóncava; escotadura amplia y cóncava; margen inferior con setas cortas; margen escotadura-punta con dientes pequeños; punta estrecha, redondeada; propodio corto, 1.2 x más ancho que largo; carpo inflado, 1.4 x más largo que ancho, con setas largas en su margen inferior; mero tan largo como ancho (Fig. 4E).

Pereópodo IV. Dactilo con tacón discreto; escotadura amplia, cóncava; punta aguzada, propodio 0.7 x el largo del carpo; tan largo como ancho; carpo con su margen ventral cubierto de setas muy largas; mero tan largo como el carpo, con setas en la mitad distal de su margen ventral (Fig. 4F). 
Pereópodo $V$. Dedo móvil con un penacho anterior de setas largas; dedo móvil del largo del fijo; propodio $0.3 \mathrm{x}$ el largo del carpo; $0.2 \mathrm{x}$ el largo que ancho; carpo 4x más largo que ancho, con setas largas en la mitad distal y cortas en la mitad basal del margen ventral; mero y carpo semejantes en largo y ancho que el propodio; ambos márgenes del carpo con setas en su mitad basal; mero setoso en ambas márgenes (Fig. 4G).

Segmentos pleonales. Coxas disminuyendo en largo hacia detrás; con setas alargadas en todos los márgenes laterales de las coxas; con setas cortas en los márgenes anterior y posterior de las coxas (Fig. 4H).

Segmento pleonal I. Margen anterior convexo; margen posterior poco cóncavo; $1.2 \mathrm{x}$ más ancho que largo (Fig. 4H).

Segmento pleonal II. Margen anterior recto; margen posterior cóncavo; márgenes posteriores muy convexos; pleuras expandidas lateralmente; 1.4 x más largo que el I (Fig. 4H).

Segmento pleonal III. Pleuras $0.4 \mathrm{x}$ el largo de las pleuras II; con el margen anterior curvado hacia delante; margen posterior cóncavo; 0.8 x del largo del II; 1.8 x más largo que el IV (Fig. 4H).

Segmento pleonal IV. Pleuras $0.7 \mathrm{x}$ del largo de las pleuras III; 0.9 x del largo del III (Fig. 4H).

Segmento pleonal $V$. Márgenes laterales oblicuos; margen posterior casi recto; 2 x más ancho que largo (Figs. 4H, 4I).

Segmento pleonal VI. Margen posterior convexo; 1.1x más ancho que largo (Figs. 4H, 4I).

Telson. Acorazonado; 1.3 x más ancho que largo; con una hilera longitudinal de setas en su cara dorsal; márgenes con setas curvadas largas laterales y distales, entre ellas, otras más cortas y rectas (Fig. 4I).

Macho desconocido.

Material estudiado. Holotipo, hembra adulta; $11 \mathrm{~mm}$ de largo del carapacho y $13 \mathrm{~cm}$ de ancho; recolectado el 12 de mayo de 2018; zona litoral; Playa Villa Rica, Veracruz, México. Depositado en la Colección Nacional de Crustáceos, Facultad de Biología, Universidad Nacional Autónoma de México, CNCR 34795.

Etimología. Especie dedicada con el mayor aprecio al Dr. Christopher B. Boyko, por sus notables contribuciones al conocimiento de los crustáceos decápodos del mundo y a los cangrejos de arena, en particular.

Distribución. Conocida solamente de la localidad tipo: Playa Villa Rica, Veracruz, México. 


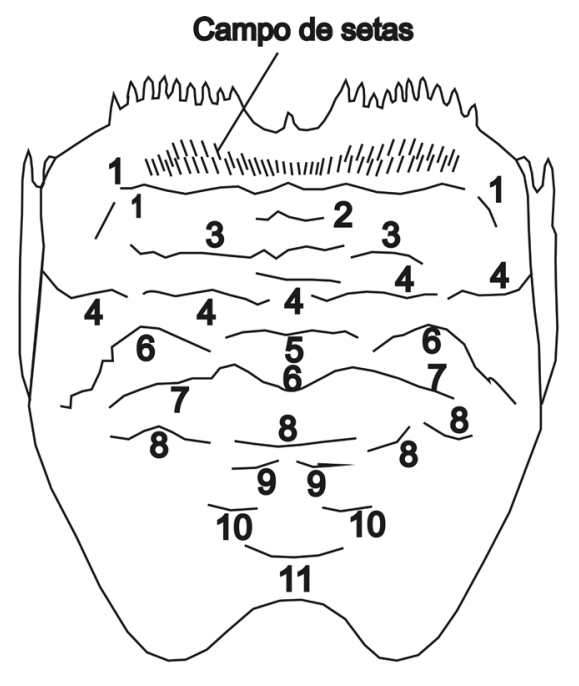

A

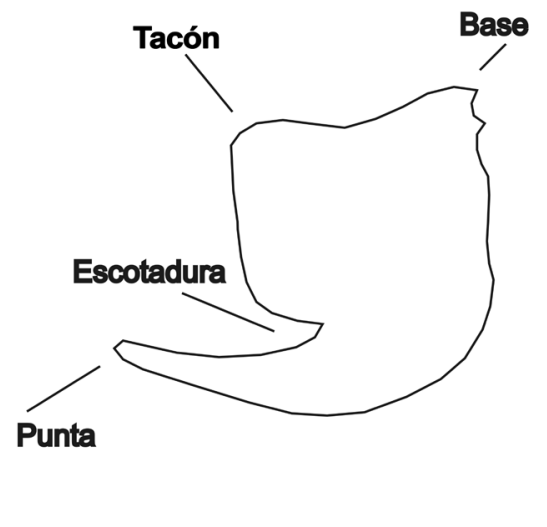

B

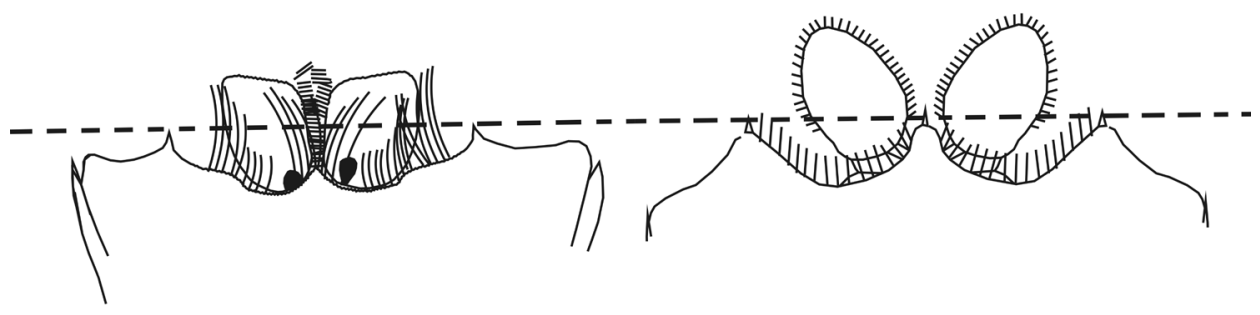

C

D

Figura 1. Terminología empleada. A, vista dorsal del carapacho de un cangrejo de arena señalando el campo de setas y los surcos del carapacho (CG 1-CG 11); B, partes del dactilo de un pereópodo I (modificado de Boyko, 2002); C, carapacho con el rostro atrasado en relación a sus espinas intermedias; D, carapacho con el rostro en línea con sus espinas intermedias. 


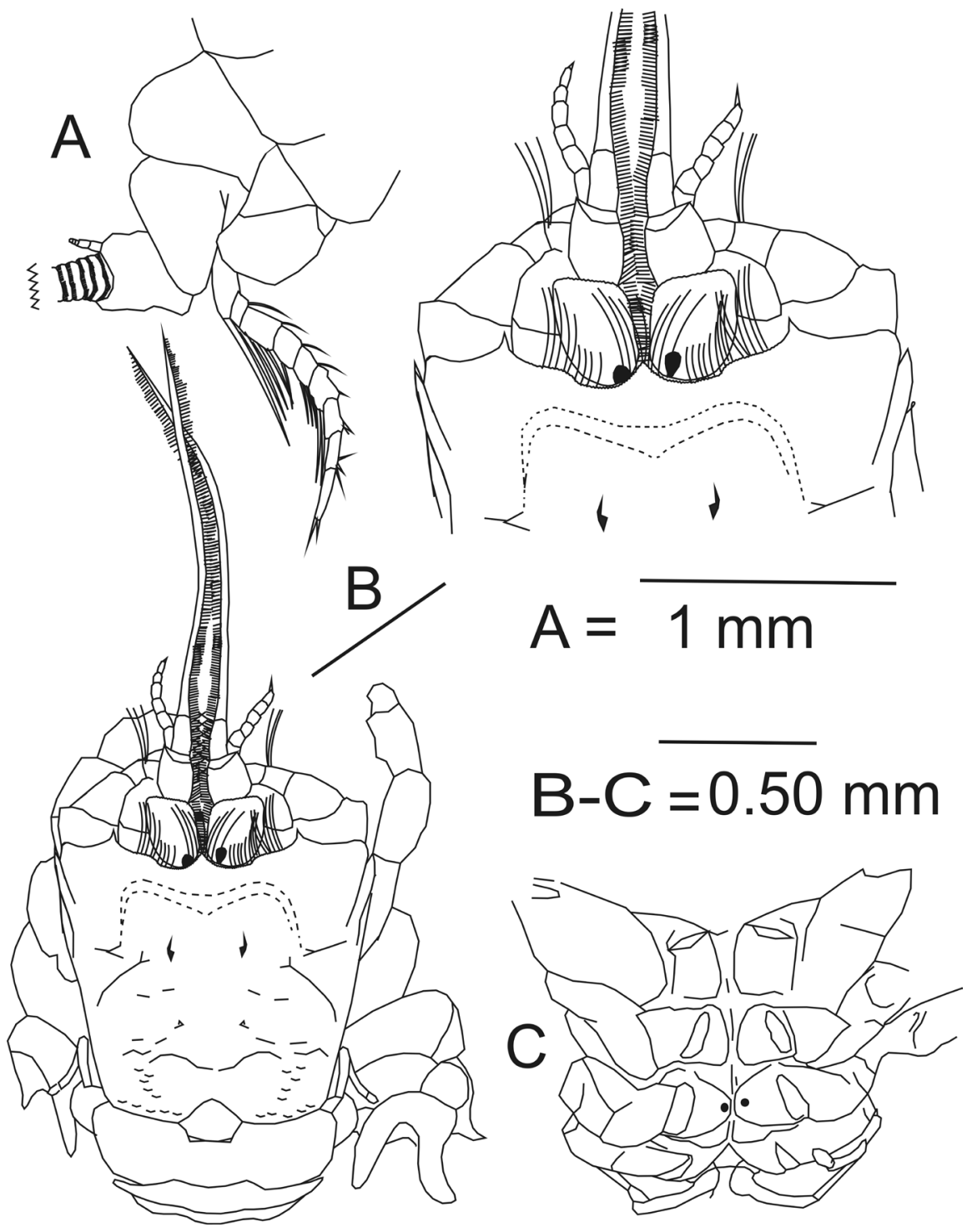

Figura 2. Lepidopa boykoi especie nueva. A, base de las antenas; B, vista dorsal del holotipo; C, vista ventral del esternón, mostrando la posición de los poros genitales femeninos. 

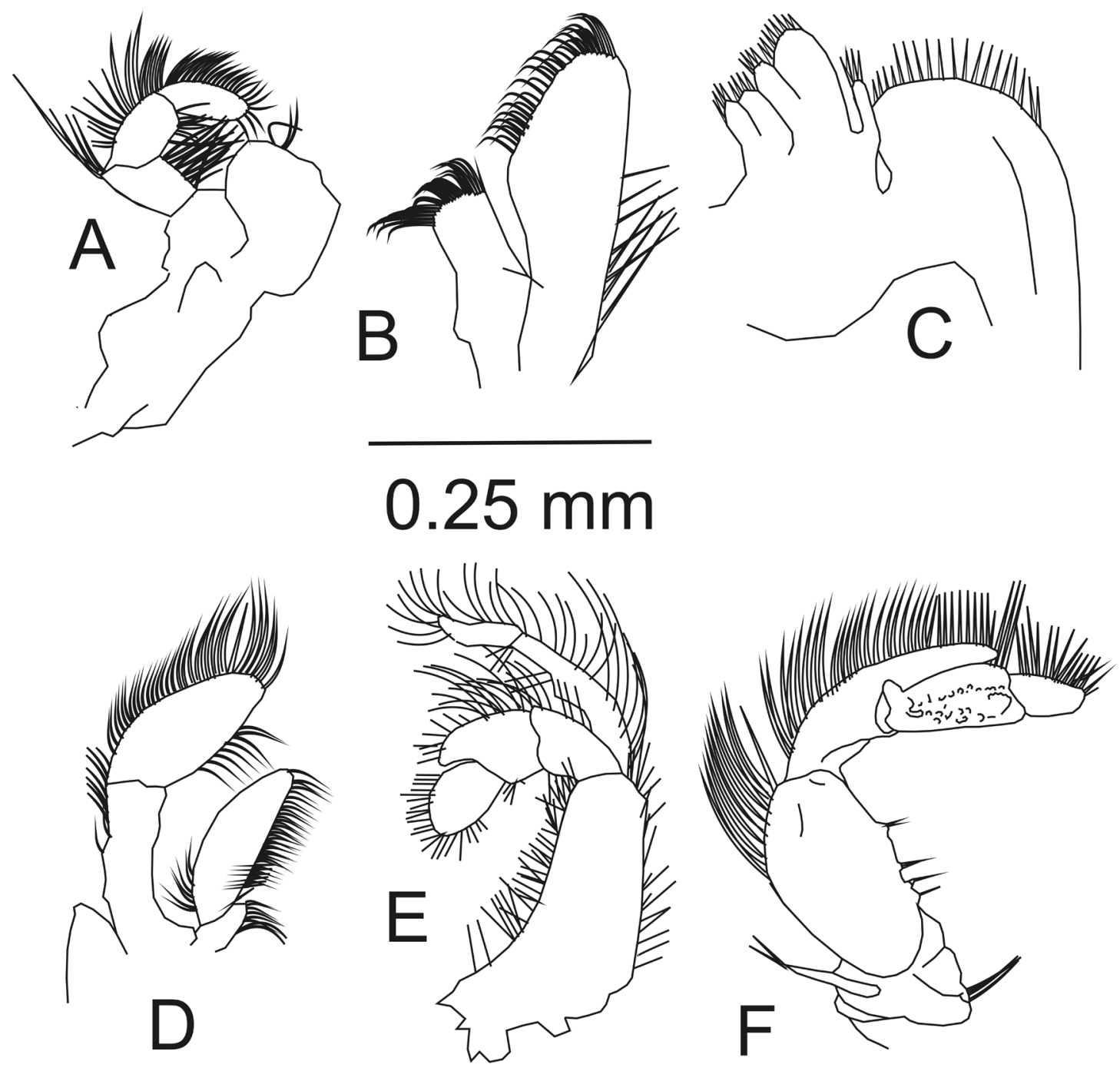

Figura 3. Lepidopa boykoi especie nueva. A, mandíbula; B, maxílula; C, maxila; D, primer maxilípedo; E, segundo maxilípedo; $\mathrm{F}$, tercer maxilípedo. 


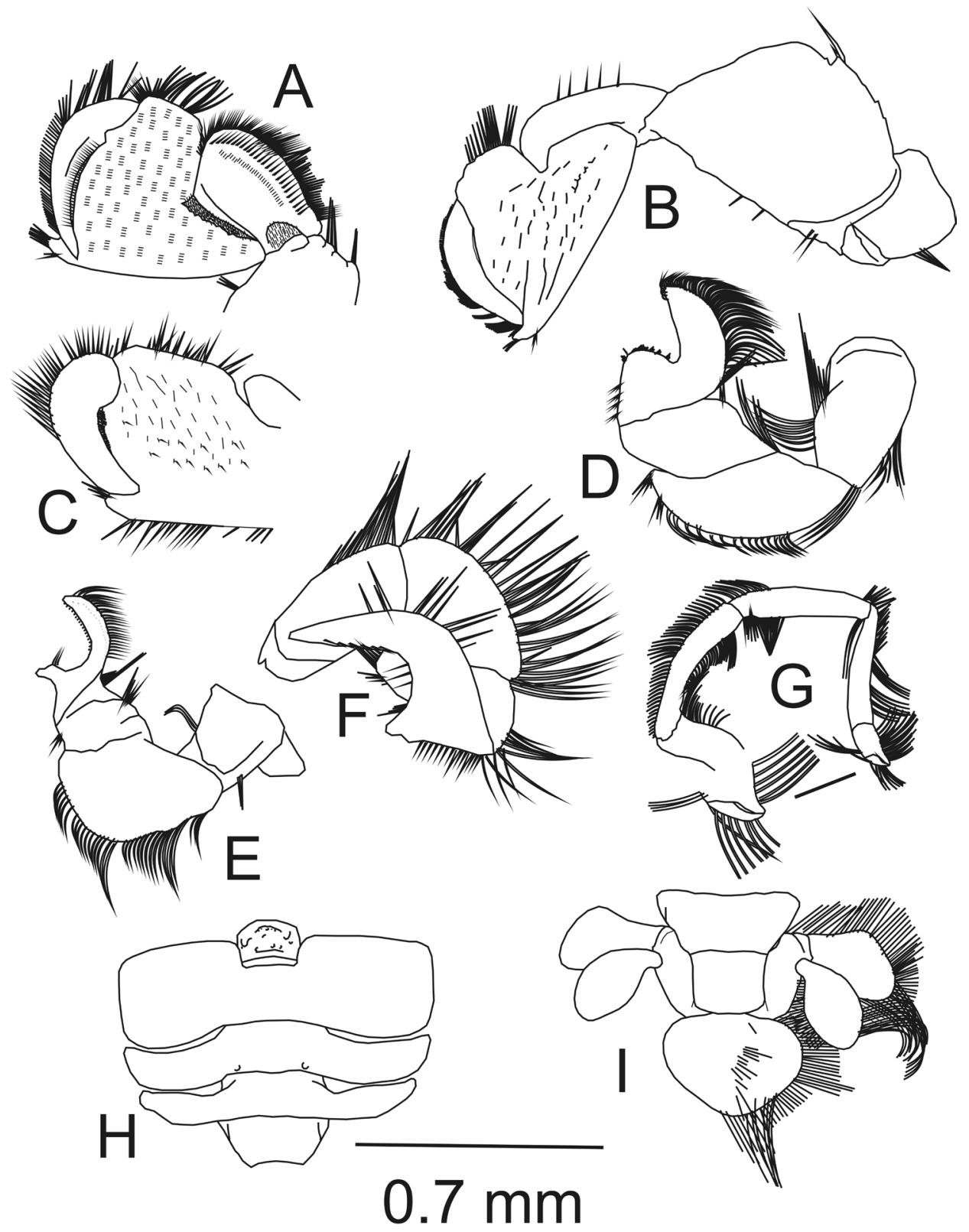

Figura 4. Lepidopa boykoi especie nueva. A, vista externa del pereópodo I derecho; B, vista interna del pereópodo I; C, vista externa del pereópodo izquierdo; D, pereópodo II; E, pereópodo III; F, pereópodo IV; G, pereópodo V; H, segmentos pleonales I-V; I, segmentos pleonales V-VI con el telson (setas del lado izquierdo eliminadas). 

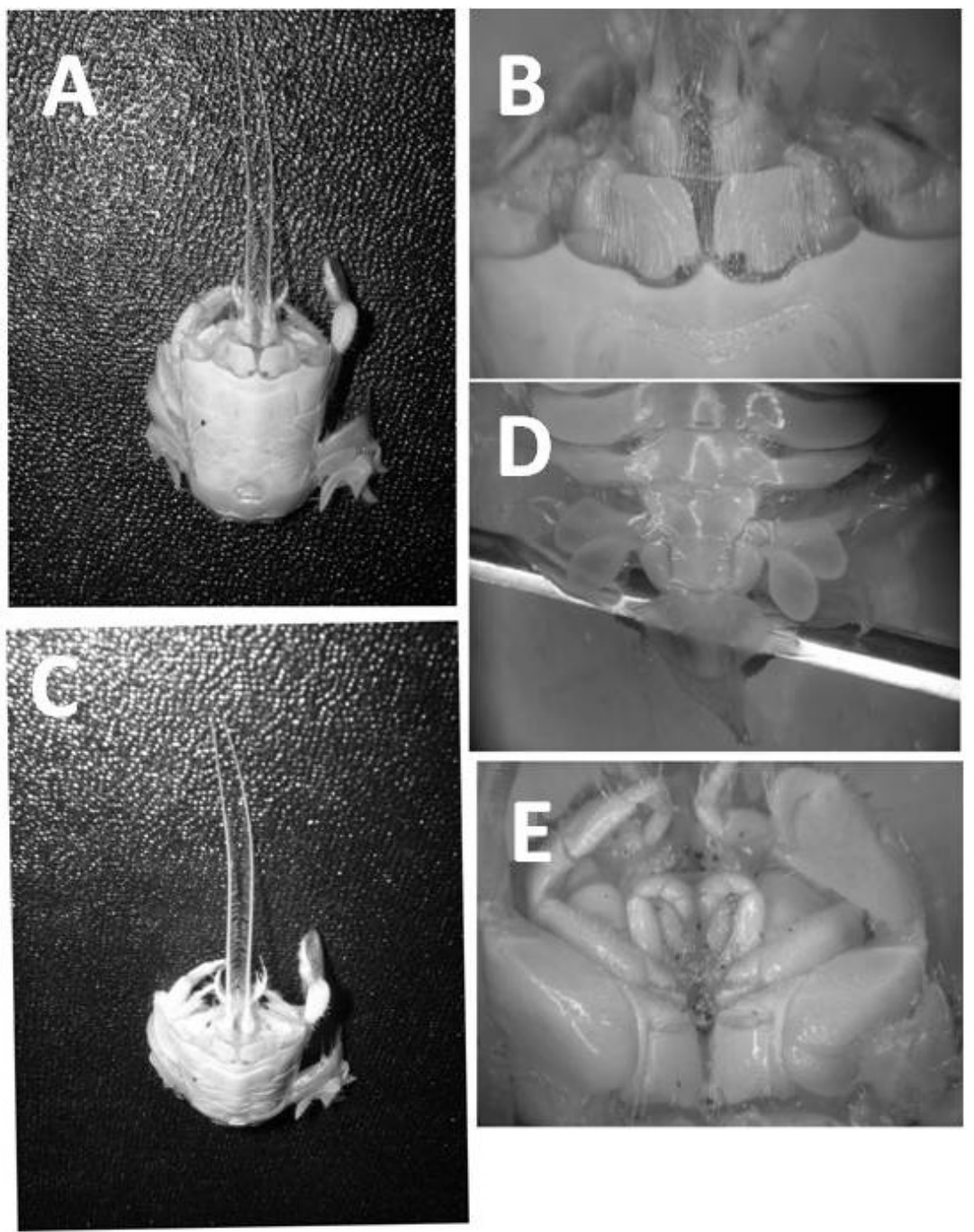

Figura 5. Lepidopa boykoi especie nueva. A, vista dorsal del holotipo; B, vista de la parte anterior del carapacho; C, vista anterior del holotipo; D, segmentos abdominales II-VI con el telson; E, vista del maxilípedo III. 


\section{CLAVE PARA LA IDENTIFICACIÓN DE LAS SIETE ESPECIES DE LEPIDOPA PRESENTES EN EL GOLFO DE MÉXICO Y EL MAR CARIBE}

1A- Borde anterior del carapacho con la punta del rostro retrasada en relación a las puntas de las espinas intermedias

1B- Borde anterior del carapacho con la punta del rostro al mismo nivel que las puntas de las espinas intermedias

2A- Pedúnculos oculares rectangulares; con córneas presentes en sus ángulos basales internos; tacón del dactilo del pereópodo I redondeado; borde ventral distal del dactilo cubierto de setas largas curvadas ------------------------------------------ L. boykoi especie nueva (Fig. 1-5)

2B- Estos caracteres no se combinan ------------------------------------------------------------------ 4

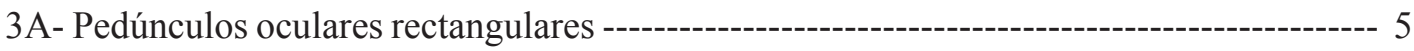

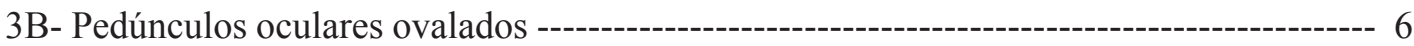

4A- Pedúnculos oculares ovalados; sin córneas presentes; tacón del dactilo del pereópodo I aguzado; borde ventral distal del dactilo con setas muy cortas ------------- L. websteri (Fig. 6)

4B- Pedúnculo oculares pentagonales; con pigmento difuso presente en el pedúnculo ocular; rostro puntiagudo, sin espina sub-marginal terminal; tacón del pereópodo 1 redondeado -----L. dexterae (Fig. 7)

5A- Pedúnculos oculares con una escotadura en su margen externo; pleuras del segundo segmento abdominal en posición oblícua, dirigidas hacia atrás ---------- L. benedicti (Fig. 8)

5B- Pedúnculos oculares sin escotadura en su margen externo; pleuras del segundo segmento abdominal en posición no oblícua, dirigidas hacia los lados ----------- L. richmondi (Fig. 9)

6A- Rostro redondeado sobre una espina sub-marginal terminal; dactilo del pereópodo 1 con el tacón puntiagudo --------------------------------------------------- L. venusta (Fig. 10)

6B- Rostro triangular; sin espina sub-marginal; dactilo del pereópodo I con el tacón redondeado L. luciae (Fig. 11) 


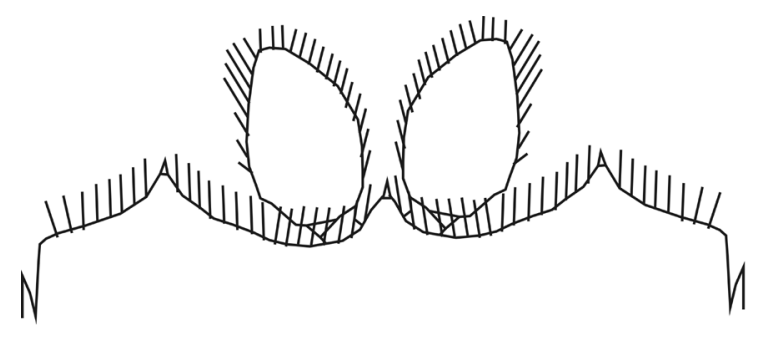

A

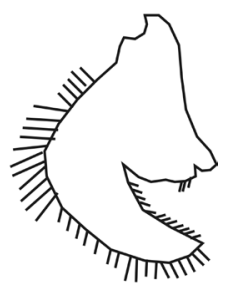

B

Figura 6. Lepidopa websteri. A, vista del margen anterior del carapacho; B, dactilo del pereópodo II.
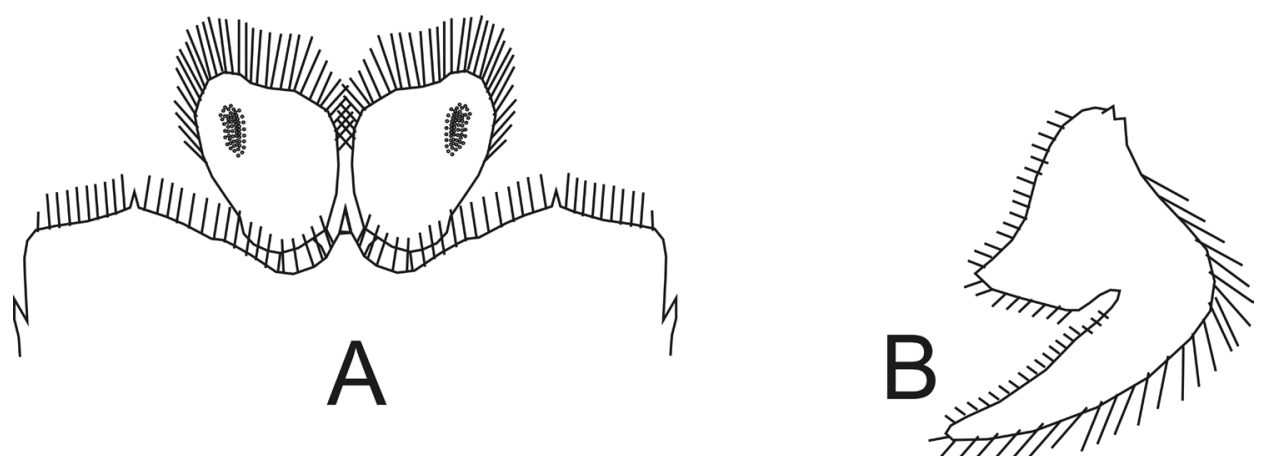

Figura 7. Lepidopa dexterae. A, vista del margen anterior del carapacho; B, dactilo del pereópodo II.

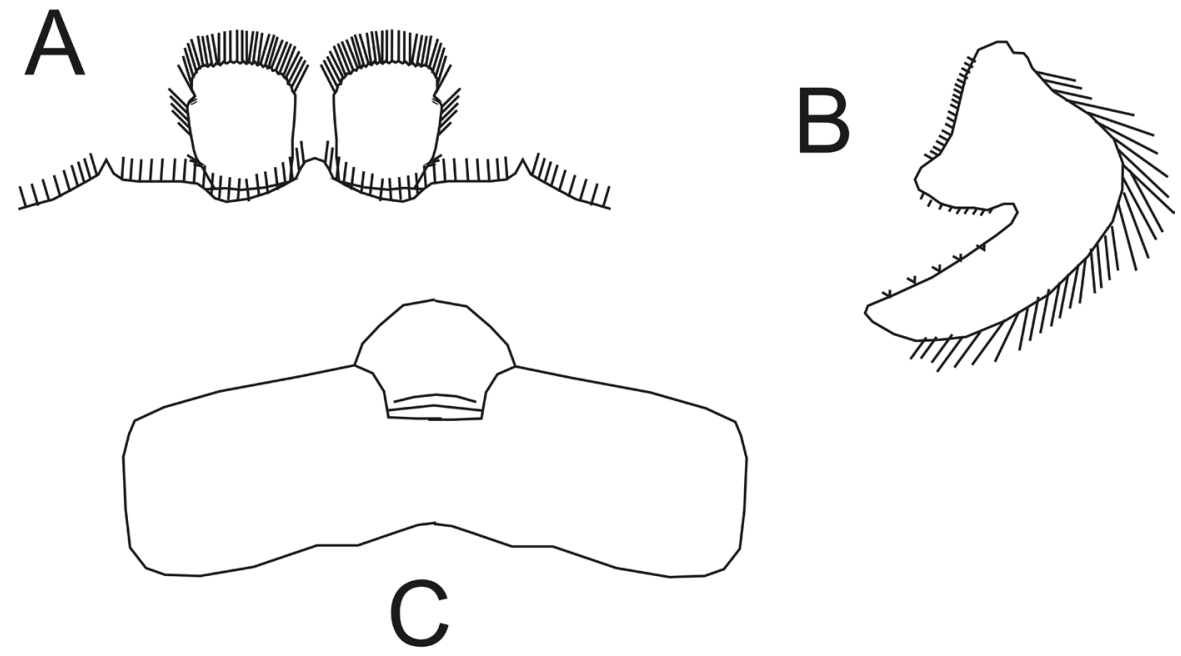

Figura 8. Lepidopa benedicti. A, vista del margen anterior del carapacho; B, dactilo del pereópodo II; C, segmento pleonal II. 

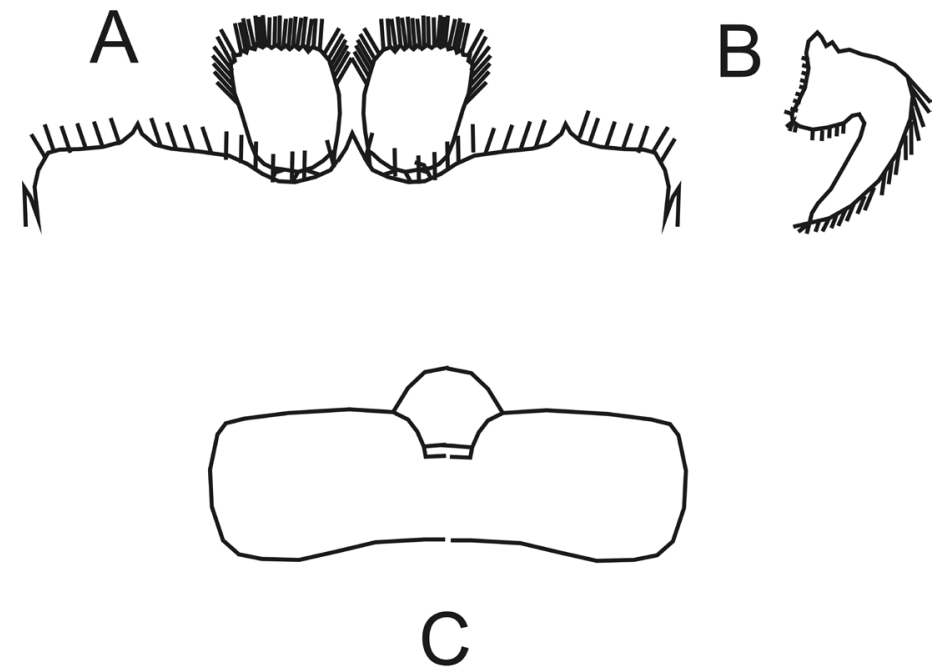

Figura 9. Lepidopa richmondi. A, vista del margen anterior del carapacho; B, dactilo del pereópodo II; C, segmento pleonal II.
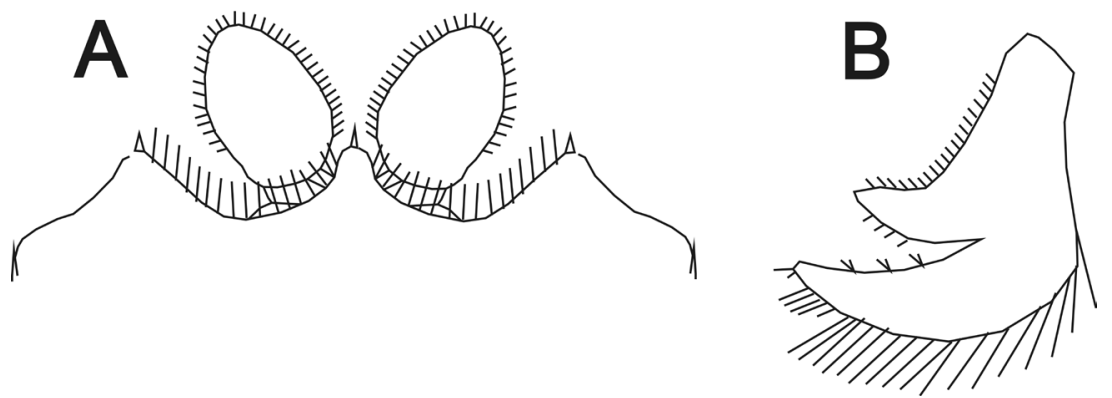

Figura 10. Lepidopa venusta. A, vista del margen anterior del carapacho; B, dactilo del pereópodo II.

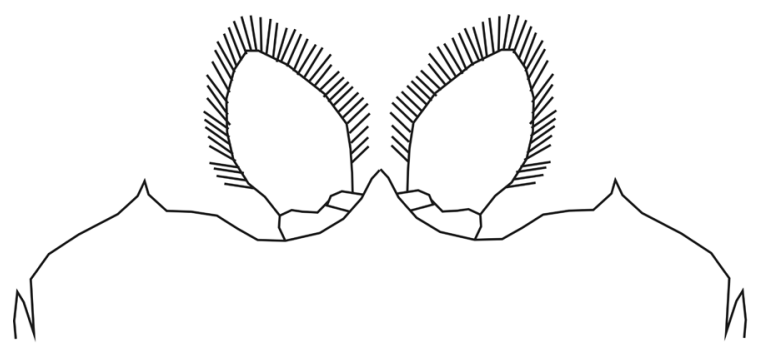

A

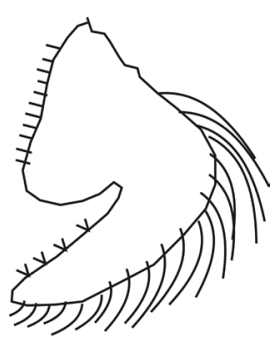

B

Figura 11. Lepidopa luciae. A, vista del margen anterior del carapacho; B, dactilo del pereópodo II. 


\section{DISCUSIÓN}

La especie nueva Lepidopa boykoi se diferencia con facilidad de las restantes especies de la región mediante el empleo de la clave ilustrada original que se presenta. Es muy afín con $L$. websteri y $L$. dexterae, ambas citadas para la región, por presentar también el rostro atrasado en relación con las espinas intermedias del margen anterior del carapacho; sin embargo, ninguna posee el segmento II del pleón con las pleuras dirigidas lateralmente. De las especies conocidas fuera del golfo de México y el mar Caribe, las únicas especies que presentan la misma relación del rostro con las espinas intemedias son L. chilensis Lenz, 1902, L. californica Efford, 1971 y $L$. deamae Benedict, 1903, pero ninguna de ellas presenta la córnea de sus ojos evidente y situada en el ángulo basal interno de sus pedúnculos, que son además rectangulares.

\section{CONCLUSIONES}

Se describe la séptima especie de Lepidopa para las aguas del golfo de México y el mar Caribe y la décima quinta para el mundo. No se descarta la posibilidad de encontrar nuevos cangrejos de arena mexicanos. Casi siempre han sido observados o recolectados durante actividades docentes, sin intenciones de clasificarlos correctamente.

\section{AGRADECIMIENTOS}

Agradecemos el envío de la muestra del cangrejo de arena a la Maestra en Ciencias Angélica Vázquez Machorro.

\section{LITERATURA CITADA}

Álvarez, F., J. L. Villalobos, M. E. Hendrickx, E. Escobar-Briones, G. Rodríguez-Almaraz y E. Campos. 2014. Biodiversidad de crustáceos decápodos (Crustacea: Decapoda) in México. Revista Mexicana de Biodiversidad Supl., 85: S208S219 DOI: 10.7550/rmb.38758.

Boyko, C. B. 2002. A Worldwide revision of the recent and fossil sand crabs of the Albuneidae Stimpson and Blepharipodidae, new family (Crustacea: ecapoda:Anomura: Hippoidea) Bulletin of the American Museum of Natural History, 272 (396): 396 pp.

Felder, D., F. Álvarez, J. W. Goy y R. Lemaitre. 2009. Chapter 57-Decapoda (Crustacea) of the Gulf of Mexico, with comments on the Amphionidacea. En: Gulf of Mexico its origin, waters and biota, biodiveresity, D. L. Felder y D. K. Camp (editores). TAMU Press, College Station. 1019-1104 pp.

Ortiz, M., F. Ocaña y R. Lalana. 2013. Clave ilustrada para identificar los cangrejos topos (Decapoda, Anomura, Hippoidea) de las aguas marinas cubanas, con un nuevo registro. Revista cubana de Ciencias Biológicas, 2 (2): 31-40.

Ortiz, M. 2015. Una nueva especie de cangrejo topo ciego del género Zygopa Holthuis, 1961 (Crustacea, Decapoda, Anomura) de Cuba. Novitates Caribaea, 8: 82-94.

Rodríguez, G. 1980. Crustáceos Decápodos de Venezuela. Instituto Venezolano de Investigaciones Científicas, $493 \mathrm{pp}$.

World Register of Marine Species (WoRMS). 2018. Available from http://www.marinespecies. org at VLIZ. Accessed 2018-06-15. doi:10.14284/170. 